\section{Gestión de una oficina de educación médica: evidencia de 10 años y planteamientos de nuevos desafíos para el siglo XXI}

\author{
LUCÍA SANTELICES ${ }^{1, \mathrm{a}, \mathrm{f}}$, CAROLINA WILLIAMS ${ }^{1, \mathrm{~b}, \mathrm{f}}$, \\ ALBERTO DOUGNAC ${ }^{1}$, SANDRA BITTNER ${ }^{2, \mathrm{c}, \mathrm{g}}$, MARCELO CÁDIZ $^{3, \mathrm{~d}, \mathrm{~h}}$, \\ ERNESTO VEGA ${ }^{1}$, MAURICIO SOTO-SUAZO ${ }^{1, e, i}$
}

\section{Retrospective analysis of the work of a medical education office}

Background: Training of health care students at universities is a great challenge for Medical Education Offices. Our office made clear and explained the teaching-learning process from the perspective of teachers, programs, and students. Aim: To report a ten years' analysis of a Medical Education Office (MEO) work, describing the different processes and systematized decisions aimed to improve the quality of the programs and learning results. Material and Methods: A 10 years retrospective analysis of the Medical Education Office processes directed to Medicine, Nursing, Physical Therapy, and Nutrition careers of a Faculty of Medicine. Flunks between 2013 and 2017 were compared. Results: A progressive reduction in flunks was observed in the four careers. Specifically, the proportion of flunks in Nutrition decreased from 30 to $9 \%$. When comparing flunks using a Chi-square test of homogeneity in the four careers, a significant decrease in four of six courses was observed. This led to a sustained increase in number of students who completed their career and obtained their title. Specifically, in Medicine there was a 7.5-fold increase in these figures. The Diploma course trained $90 \%$ of the teachers in charge of courses of the four careers. The master's degree generated research that allowed to increase the productivity in health sciences education. Conclusions: The Office of Medical Education created knowledge and management models for the education of health sciences students, enhancing the quality of training and learning processes.

(Rev Med Chile 2021; 149: 119-127)

Key words: Education, Medical; Education, Medical, Undergraduate; Learning; Teaching.

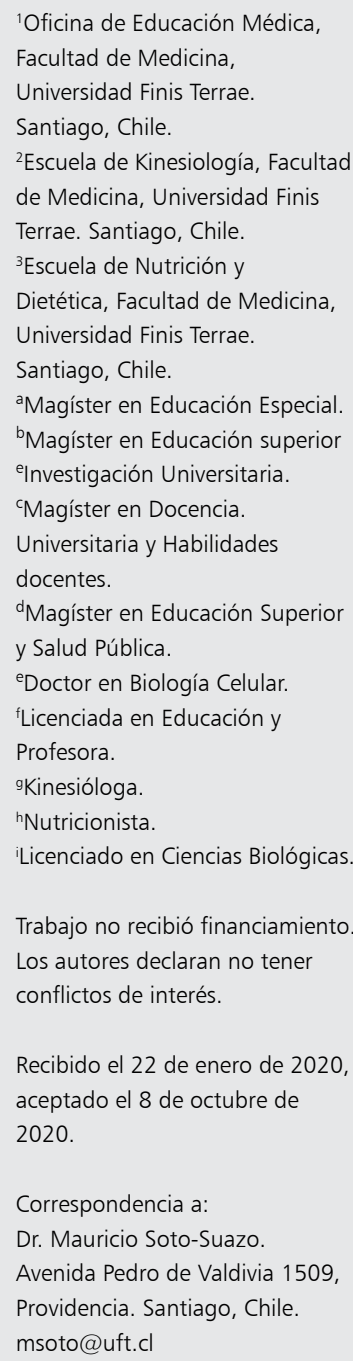

\section{L}

a exigencia de planificar situaciones de enseñanza-aprendizaje contextualizadas, y manejar la gestión curricular ${ }^{1}$ como "un conjunto de decisiones que se deben asumir para planificar, poner en marcha, y evaluar el proceso de enseñanza-aprendizaje, así como el estudio teórico que lleva a su comprensión" desafía a las oficinas de educación médica y ciencias de la salud (OFEMyCS) a:
- Mejorar la calidad de los programas de formación en pregrado y apoyar estos avances con evidencias ${ }^{3}$.

- Fortalecer elementos formativos en los docentes para que interactúen pedagógicamente con el estudiante ${ }^{4,5}$.

- Formar profesionales de la salud competentes, con sólidos conocimientos científicos, com- 
prometidos moralmente con las personas y con las funciones que les corresponderá asumir ${ }^{6}$, con las características de los jóvenes que hoy acceden a la educación superior ${ }^{7,8}$.

La gestión de las Oficinas de Educación Médica y Ciencias de la Salud (OFEMyCS) ha incorporado los currículos formativos de otros profesionales del área de la salud, lo que hace más complejo orientar el trabajo de esta instancia. A pesar del esfuerzo e iniciativas para abordar esta realidad, los resultados de esos procesos han sido documentados parcialmente desde la investigación, lo que no permite replicar el camino recorrido ${ }^{9}$.

En virtud de lo señalado surgió una interrogante: ¿Cómo establecer una ruta de procesos y toma de decisiones que dé cuenta de un modelo de gestión académica de una OFEMyCS que asume los cambios constantes que experimentan los currículos, los docentes y los estudiantes en el proceso formativo en carreras de salud?. El objetivo de este artículo fue relatar la experiencia de los procesos y decisiones sistematizados durante 10 años por la Oficina de Educación Médica de la Facultad de Medicina de la Universidad Finis Terrae (OFEM), que presentó mejoras sustantivas en la calidad de los programas, de los docentes y de los resultados de aprendizaje de los estudiantes.

La experiencia consideró un tiempo de observación y acumulación de evidencias por diez años. Se concretó en las escuelas de Medicina, Enfermería, Nutrición y Dietética y Kinesiología.

La trayectoria se inició con la planificación estratégica de la facultad que identificó tres factores críticos de éxito académico:

1. La calidad de los estudiantes que ingresan.

2. La calidad del currículo impartido.

3. La calidad de los docentes que participan en el proceso.

El plan de acción se inició con el diseño de un marco de referencia que gestó una línea de base teórica para contrastar las evidencias reunidas y tomar decisiones en torno a los tres factores críticos mencionados ${ }^{10}$.

Para reunir evidencias acerca de la aplicación del plan de acción se asoció, a cada factor, líneas de investigación formativa ${ }^{11}$.

- Línea asociada a estudiantes: Se estudiaron variables del perfil de ingreso (ponderaciones, conocimientos escolares); correlación entre variables de ingreso y rendimiento académico; análisis de factores de predictibilidad de éxito académico; evolución de tasas de deserción y del rendimiento académico en asignaturas iniciales; y evaluación del cumplimiento de los perfiles de egreso.

- Línea asociada al currículo: Se analizaron los perfiles de egreso, la pertinencia de los planes de estudio y la coherencia entre contenidos, metodologías y sistemas de evaluación de los programas de asignaturas. Además, se diseñó un plan de seguimiento del cumplimiento de los objetivos de aprendizaje. Se realizó monitoreo de conducción de clases realizadas en aulas y clínicas.

- Línea asociada a docentes: Se analizó el impacto de la capacitación docente y comunidades de aprendizaje sobre logro de aprendizajes de los estudiantes.

Desde cada uno de los factores surgieron propuestas a corto, mediano y largo plazo. Las propuestas dieron origen a un plan de acción que se describirá a continuación.

\section{Calidad de los estudiantes}

\section{Objetivo}

El objetivo final de este factor fue fomentar el éxito académico de los estudiantes que ingresan a la Facultad de Medicina independiente de su perfil de ingreso.

\section{Diagnóstico}

Las altas tasas de reprobación observadas en los dos primeros semestres de cada carrera, asociadas a la existencia de vacíos de conocimientos científicos de los estudiantes admitidos, obligaron a considerar intervenciones sobre ellos.

\section{Plan de acción}

\section{Hito 1: Formación de equipos académicos y comunidades de aprendizaje}

Para trabajar con las necesidades de estudiantes de ingreso y recabar las evidencias de ese momento (2010-2011), se formaron equipos académicos. Cada escuela invitó a docentes que se reunieran al menos dos veces al mes para discutir y tomar decisiones con respecto a los conocimientos definidos como requisitos necesarios para enfrentar el primer año de la carrera. Apoyados por OFEM, 
se revisaron los contenidos de la enseñanza secundaria junto a contenidos del primer semestre de universidad, posteriormente, se diseñó y validó un instrumento de evaluación diagnóstica $(\text { EVALD })^{12}$.

\section{Hito 2: Programa de nivelación de conocimientos} científicos requisitos

Se diseñó, implementó y evaluó un programa de nivelación, el cual fue incorporado como un proceso continuo. Las escuelas de Medicina, Enfermería, Kinesiología y Nutrición, apoyados por OFEM, revisaron los contenidos de las asignaturas del primer semestre y los compararon con los contenidos de la educación media. Producto de este análisis, se diseñó y validó un instrumento de evaluación diagnóstica (EVALD) de conocimientos requisitos para estudiantes de ingreso ${ }^{12}$.

Se diseñó el programa de nivelación que se inicia con el proceso de matrícula, el estudiante rinde la EVALD, y, al mismo tiempo, recibe un manual de estudio que contiene los conocimientos científicos que son requisitos para sus asignaturas de primer semestre. Posteriormente, asiste a clases presenciales, por un total de $25 \mathrm{~h}$ cronológicas, las que tienen como base el manual.
Durante el primer año lectivo, con la finalidad de disminuir la reprobación de los estudiantes, se usaron los resultados obtenidos por EVALD del programa de nivelación y las primeras evaluaciones de las asignaturas del primer semestre, con ello se detectó a los estudiantes en riesgo académico. Se les apoyó con acciones remediales: entrevistas, clases remediales, intervenciones psicopedagógicas, acompañamiento del estudiante. Los resultados de EVALD mostraron ser predictores de rendimiento académico.

Con cinco años de ejecución de este programa de nivelación, el impacto ha evidenciado una disminución considerable de los porcentajes de reprobación en las asignaturas del primer año en las cuatro carreras (Figura 1).

Se ha observado cómo disminuye la proporción de reprobación a través del tiempo, siendo menor a 20\% en Enfermería, Kinesiología y Nutrición y menos del10\% en Medicina. Se observó que la disminución en los porcentajes de reprobación estaba directamente relacionada con la retención y avance curricular de los estudiantes. Esta acción provocó un aumento en el número de estudiantes que terminaron sus estudios y obtuvieron su título; las Figuras 2 y 3 ilustran lo señalado.

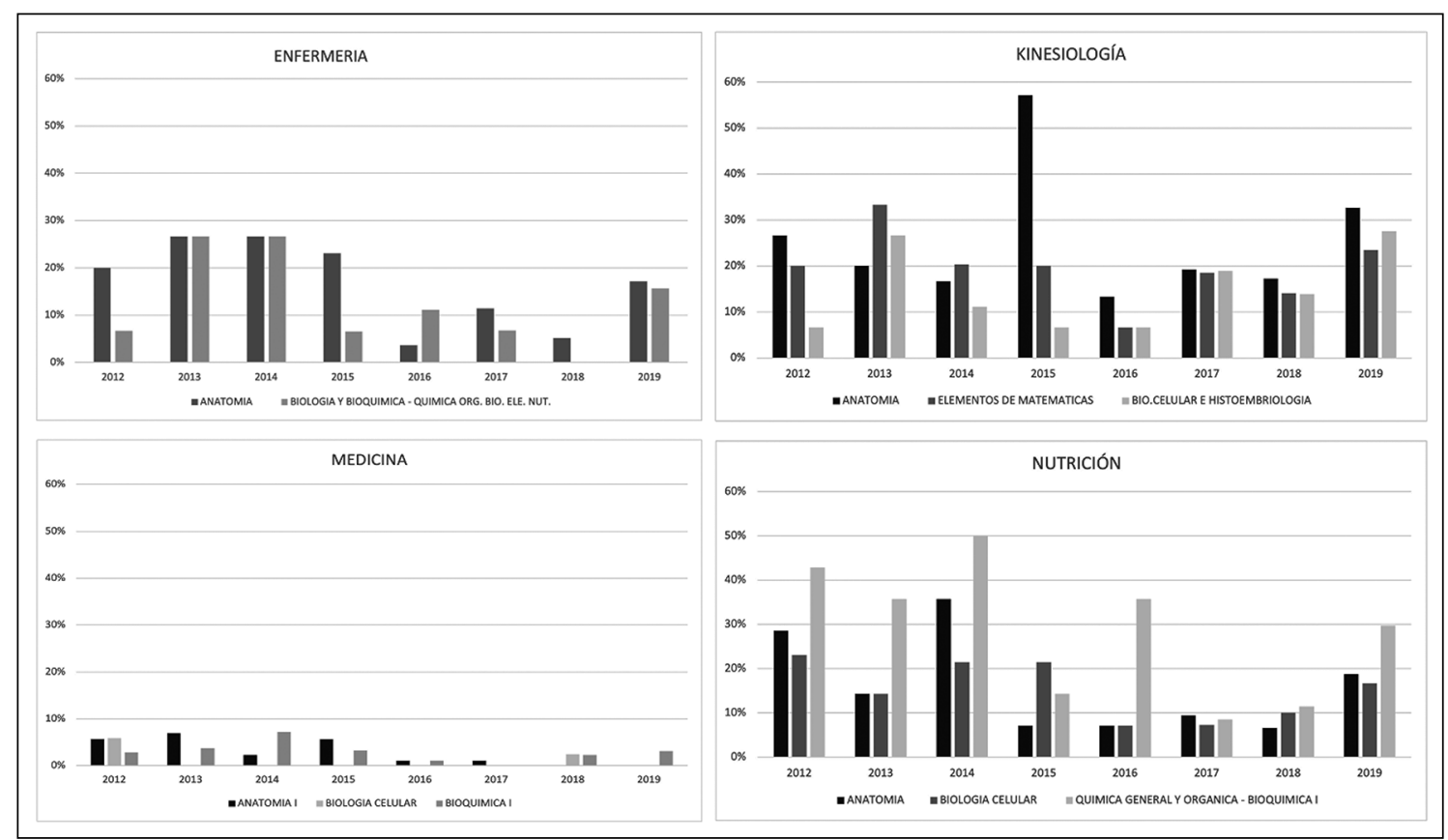

Figura 1. Tasa de reprobaciones de asignaturas del primer semestre de las carreras de Enfermería Kinesiología, Medicina y Nutrición cohortes 2012-2019. 


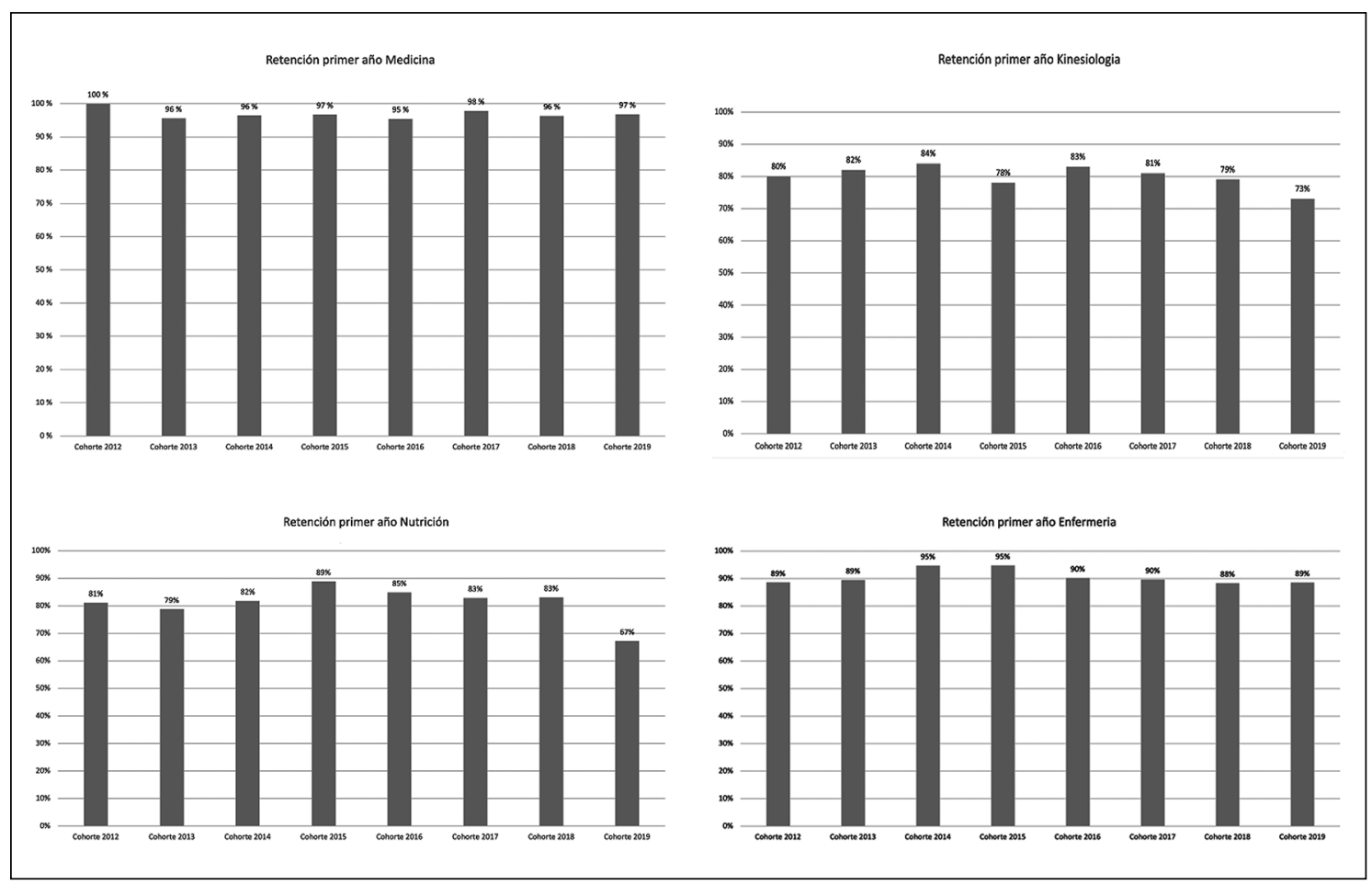

Figura 2. Retención de primer año de las carreras de Medicina, Kinesiología, Nutrición y Enfermería de las cohortes 2012-2019.

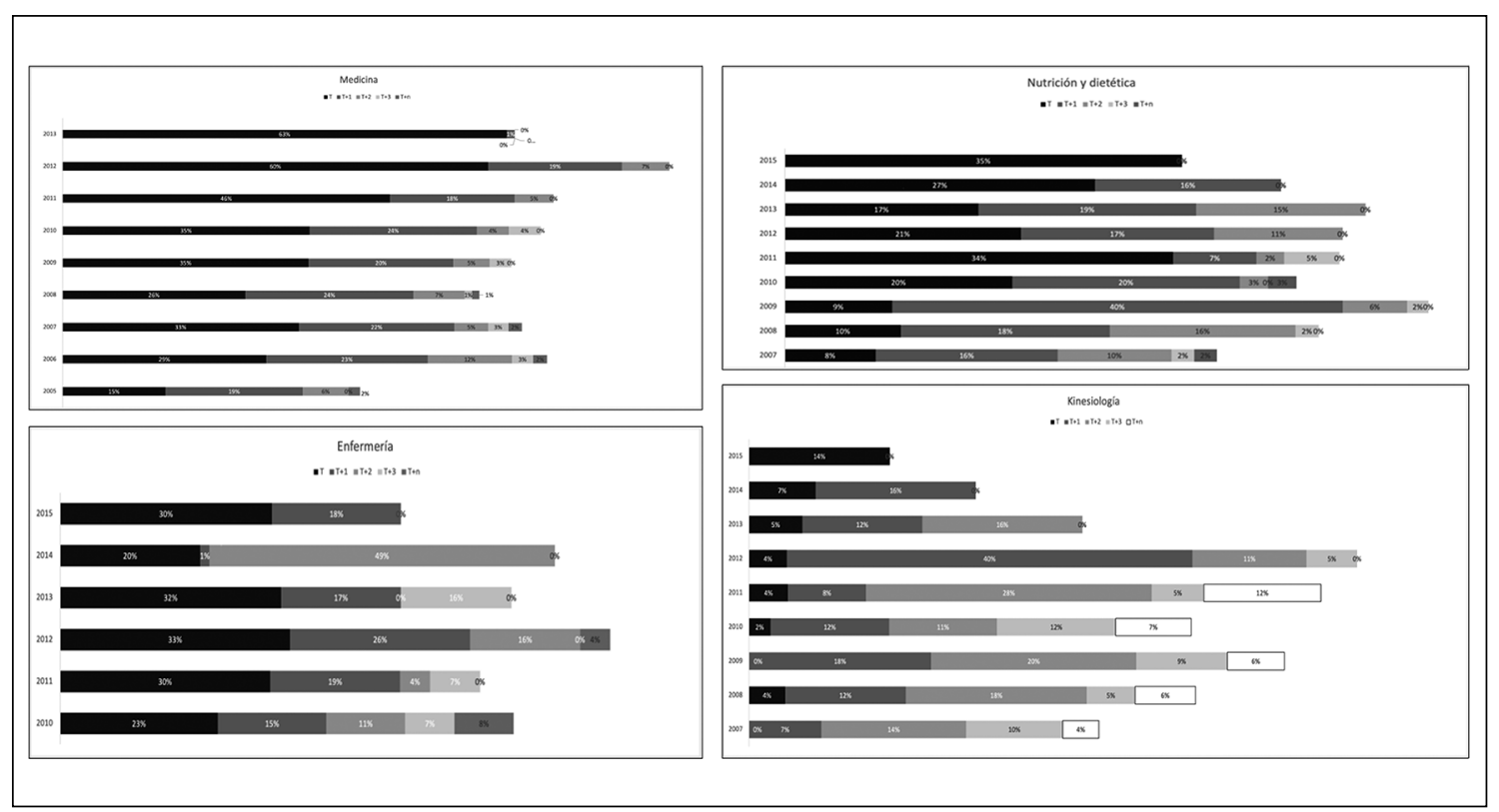

Figura 3. Titulación oportuna y efectiva de las carreras de Kinesiología, Medicina, Nutrición y Enfermería cohortes 2007-2015. 
Durante el período estudiado, Medicina tuvo una media de $96,8 \%$, Kinesiología 80\%, Nutrición $81,1 \%$ y Enfermería 90,6\%.

Se observó el aumento de la titulación efectiva de Medicina desde $40 \%$ a $86 \%$, Nutrición desde $38 \%$ a $51 \%$, Enfermería de $64 \%$ a $70 \%$ y Kinesiología de $31 \%$ a $60 \%$.

\section{Hito 3: Sistema de evaluación del seguimiento del perfil de egreso}

Para evaluar el cumplimiento de los objetivos educacionales del plan de estudios, se implementó un modelo de evaluaciones que consideró un sistema de evaluaciones diagnósticas, intermedias y finales, que dieron cuenta del avance de las competencias del perfil de egreso (Figura 4).

Tal decisión llevó a un análisis de coherencia que exigía la revisión de cada programa de asignatura y su articulación vertical y horizontal en cada uno de los planes de estudio de cada carrera. La articulación vertical da cuenta de la coherencia y exigencia de los objetivos educacionales desarrollados por cada asignatura según el nivel académico del estudiante, mientras que la articulación horizontal permite observar áreas de formación disciplinar que diferentes asignaturas articulan entre sí, permitiendo seguir su progreso en la adquisición de competencias del perfil de cada carrera.

\section{Calidad del currículo}

\section{Hito 4: Revisión y ajuste curricular}

\section{Objetivo}

Analizar los programas del plan de estudio en función de la coherencia entre el perfil de egreso y su articulación curricular a nivel vertical y horizontal.

\section{Diagnóstico}

Los análisis de los programas permitieron realizar un diagnóstico específico de elementos que todo programa bien estructurado debe presentar (Santelices et al, 2015) ${ }^{14}$. Estos elementos corresponden a una descripción breve de los propósitos de la asignatura, objetivos específicos o resultados de aprendizajes, organización de las unidades de

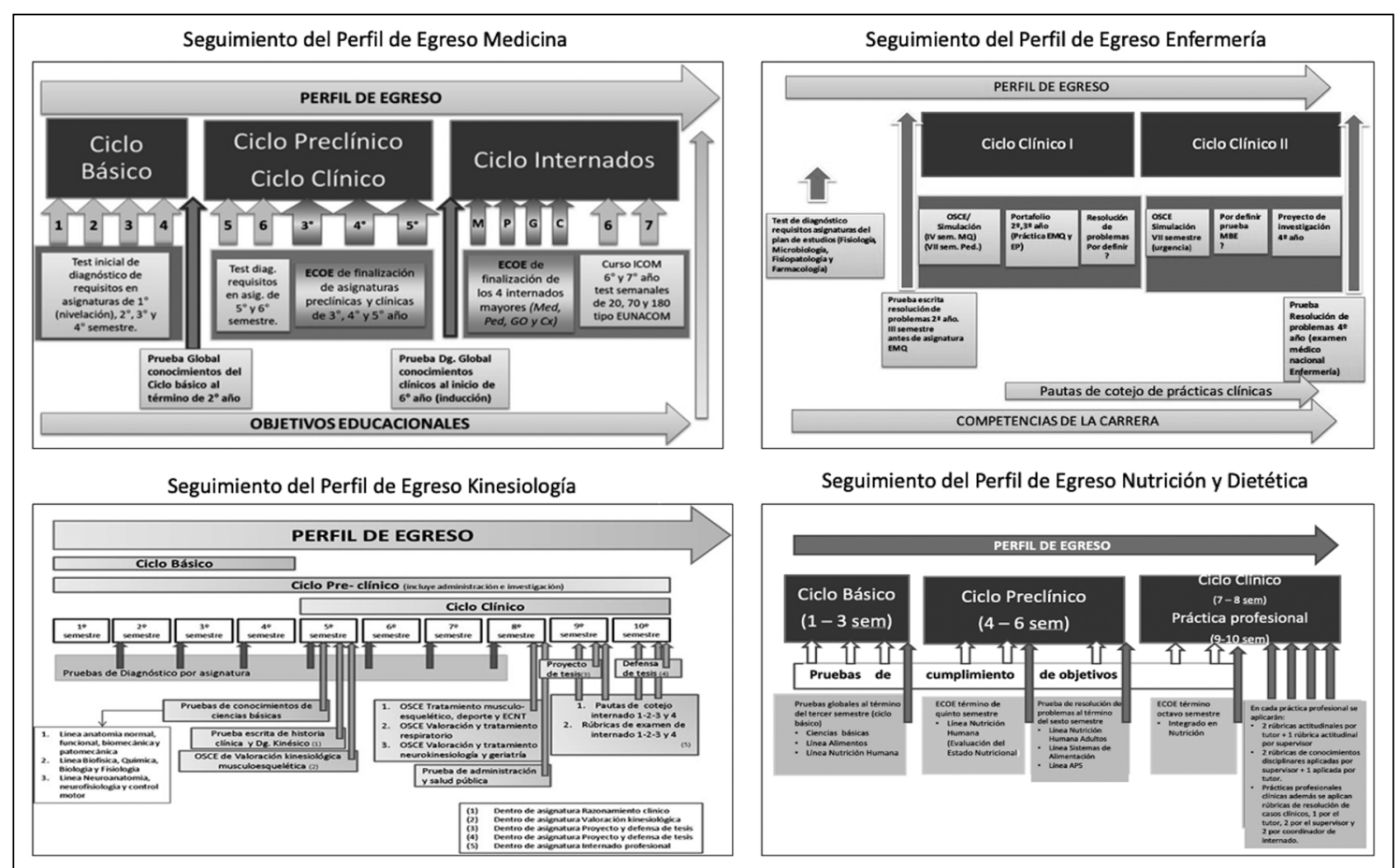

Figura 4. Modelo de seguimiento del perfil de egreso utilizados por las carreras de Medicina, Enfermería, Kinesiología y Nutrición. 
contenidos, la metodología de enseñanza y las formas e instrumentos de evaluación.

\section{Plan de acción}

A nivel curricular, se trabajó la articulación horizontal y vertical de los programas de cursos de cada carrera, para integrar los contenidos disciplinares entre sí y el nivel de las habilidades estimuladas en forma progresiva, estableciéndose una secuencia de complejidad cognitiva desde el recuerdo de información hasta el nivel de resolución de problemas en los distintos niveles formativos.

Se ajustaron las metodologías de enseñanza en función de la exigencia de cada resultado de aprendizaje esperado, junto con ello, se modificó el diseño de los instrumentos de evaluación incorporándose las tablas de especificaciones de cada instrumento.

\section{Calidad de los docentes}

\section{Objetivos}

- Preparar metodológicamente a los docentes mediante un proceso de perfeccionamiento continuo, para estimular los objetivos educacionales, por nivel, mejorando la retención y promoción de los estudiantes.

- Fortalecer la sinergia entre los docentes responsables de las diferentes líneas de formación para generar comunidades de aprendizaje y colaboración mutua.

- Estimular la participación de los académicos para colaborar con el equipo directivo, aportando información para la toma de decisiones.

\section{Diagnóstico}

En el hito 4 se realizó la detección de necesidades de perfeccionamiento en docencia, mediante un cuestionario abierto y una entrevista individual. Surgieron como temáticas relevantes: evaluación, metodologías, conducción de la docencia, estrategias de aprendizajes, y estructuración de programas de asignatura. A la luz de este diagnóstico se gestó un plan de acción.

\section{Plan de acción \\ Hito 5: Diseño y ejecución de perfeccionamiento docente \\ A raíz de este diagnóstico, se diseñó un progra- ma de perfeccionamiento en docencia universita-}

ria en ciencias de la salud con cinco módulos de aprendizajes: a) Programa de curso; b) Planificación de la docencia; c) Conducción de la docencia;

d) Estrategias de enseñanza para el aprendizaje y

e) Evaluación de los aprendizajes.

En la actualidad, los encargados de asignatura que han rendido con éxito el programa de perfeccionamiento docente son en Medicina $84 \%$, Enfermería 70\%, Kinesiología 70\% y Nutrición 67\% (Figura 5).

La medición del impacto de este diplomado reveló que el aprendizaje de nuestros estudiantes ha mejorado como consecuencia de la continuidad del programa de perfeccionamiento docente y de la difusión de buenas prácticas ${ }^{14}$. Dado la importancia de este tipo de perfeccionamiento, esta capacitación fue incorporada al perfil docente que requiere la Facultad de Medicina.

En virtud del interés en profundizar la formación en el área, surgió en el año 2014 el Magíster en Docencia Universitaria en Ciencias de la Salud, del cual, además, se generó un foco académico orientado a la investigación formativa en Educación en Ciencias de la Salud que ha reforzado los factores iniciales de esta experiencia.

\section{Hito 6: Magíster en Docencia Universitaria en Ciencias de la Salud (MDUCS)}

El MDUCS abarca áreas de investigación y desarrollo generadas a partir de temáticas emergentes, desde los ejes estratégicos de la facultad y de intereses propios de la trayectoria profesional de sus académicos:

- Aprendizaje y desempeño académico de los estudiantes.

- Fortalecimiento pedagógico de docentes.

- Las variables curriculares y sus repercusiones.

Esta última línea estudia el currículo en carreras de ciencias de la salud. Se incorporan los análisis de perfiles de egreso, y la coherencia de estos con metodologías docentes y de evaluación, creando procesos sistemáticos de seguimiento y gestión del currículo.

Producto de estas líneas de investigación se están gestando aportes al conocimiento. Algunos resultados ilustrativos se presentan en la Tabla 1.

Hasta el Hito 6 se observaron tres consideraciones: 1) La detección temprana de riesgo académico, junto a la implementación de un plan de mejora y acciones remediales; 2) La revisión y 


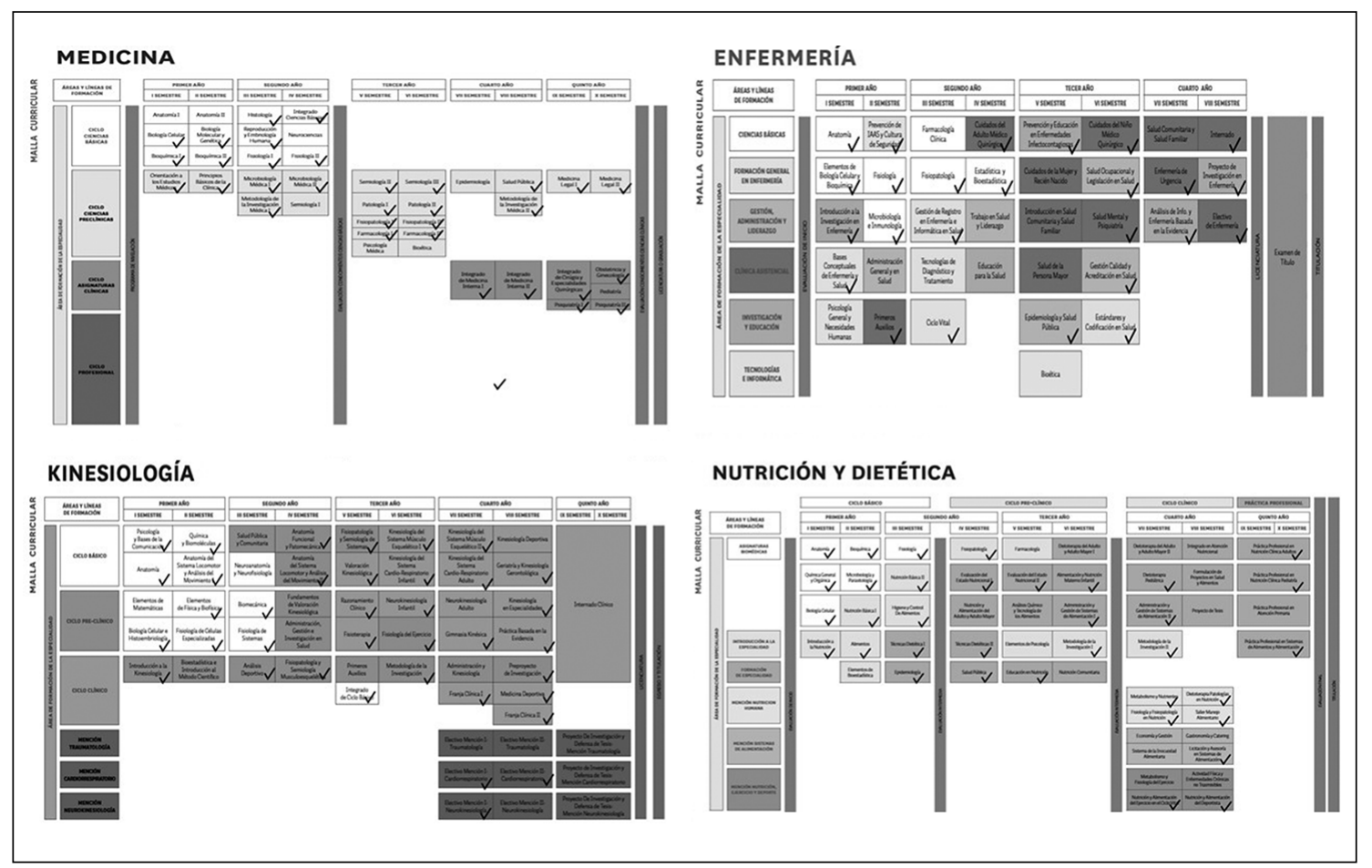

Figura 5. Cobertura del perfeccionamiento docente $(\sqrt{ })$ de los encargados de las asignaturas que componen los planes de estudios en las carreras de Medicina, Enfermería, Kinesiología y Nutrición.

Tabla 1. Ejemplos de productividad generada por las líneas de investigación del Magíster en docencia universitaria en Ciencias de la Salud

\begin{tabular}{|c|c|}
\hline Título & Revista/Congreso \\
\hline $\begin{array}{l}\text { "Stimulation of clinical reasoning in undergraduate dental Student } \\
\text { using a pedagogical intervention in dental radiology" }\end{array}$ & $\begin{array}{l}22 \text { International Congress IAD/MFR. Philadelphia } \\
\text { USA Agust 24/2019 }\end{array}$ \\
\hline $\begin{array}{l}\text { Aplicación del aprendizaje basado en problemas en estudiantes de } \\
\text { segundo año de la carrera de nutrición y dietética de la Universidad } \\
\text { Finis Terrae }\end{array}$ & $\begin{array}{l}\text { Revista Fundación de Educación Médica: FEM 2019; } \\
22 \text { (2): 85-90. }\end{array}$ \\
\hline "Efectividad de la formación en tutoría clínica en nutrición" & Revista Chilena de Nutrición 2019; 46 (3): 271-278 \\
\hline "Razonamiento clínico en Kinesiología y su aplicación docente" & Revista del Colegio de Kinesiólogos 2017, (1); 1-8 \\
\hline $\begin{array}{l}\text { Impacto de dos instrumentos de evaluación en el rendimiento } \\
\text { académico y su relación con los estilos de aprendizaje en } \\
\text { estudiantes de Kinesiología }\end{array}$ & $\begin{array}{l}\text { Revista Educación Ciencias de la Salud 2017; } 14 \\
\text { (2): 130-7 }\end{array}$ \\
\hline $\begin{array}{l}\text { Efectividad de la simulación en la educación médica desde la } \\
\text { perspectiva de seguridad de pacientes }\end{array}$ & Revista Médica de Chile 2017; 145: 514-526 \\
\hline $\begin{array}{l}\text { Efecto del Aula Volteada en el rendimiento académico: Estudio } \\
\text { comparativo basado en el resultado del rendimiento académico con } \\
\text { metodología Aula Volteada y Clase Tradicional para la asignatura de } \\
\text { Salud Pública }\end{array}$ & $\begin{array}{l}\text { Revista Educación Ciencias de la Salud 2016; } 13 \\
\text { (1): 15-20 }\end{array}$ \\
\hline
\end{tabular}


estructuración de los programas de asignaturas junto a un trabajo colegiado que ha generado una nueva cultura curricular y 3) Un proceso de formación continua de los académicos.

\section{Consideraciones finales}

Este estudio indica que, en la actualidad, las oficinas de Educación Médica o de Ciencias de la Salud requieren una nueva mirada, más allá de la transmisión de conocimientos pedagógicos generales y de evaluación tradicionales, una visión sistémica desde la comprensión y perspectiva de la gestión del currículo.

El currículo es un concepto que se confunde con la concepción de plan de estudio, sin embargo, se trata de un concepto polisémico que implica todas las variables que entran en juego desde que se toma la decisión de diseñar e implementar una carrera universitaria. Paulina Berríos ${ }^{16}$ indica la posibilidad de modificar la crítica que existe sobre la calidad de los procesos de formación profesional en carreras en ciencias de la salud realizando una acción sistemática de la gestión que responda al plan estratégico de una facultad de medicina o de ciencias de la salud, mediante una oficina de educación médica que planifique una gestión metódica sobre los tres ejes trascendentales del currículo: plan de estudio, estudiantes y docentes.

Estudios a nivel mundial y en Chile han tratado de ofrecer información empírica sobre variables vinculadas con la calidad académica ${ }^{17,18}$, tales como rendimiento académico o deserciones estudiantiles. Existe poca evidencia sistemática acerca de procesos que subyacen a la ejecución y seguimiento de los planes de estudio y por tanto, a un aspecto de gestión curricular vinculado con la calidad académica. El estudio indica que existe un conjunto de procesos asociados a la gestión del currículo que, correctamente implementados y abordados armoniosamente, modifican positivamente los resultados académicos y mejoran la calidad de la educación impartida, provocando cambios culturales en la institución ${ }^{14}$.

Para algunas autoridades universitarias y docentes, el principal determinante de altas tasas de deserción y bajos rendimiento en carreras de la salud conciernen a la baja preparación de los estudiantes desde la enseñanza media. Sin embargo, este pensamiento da cuenta de un débil análisis de las variables que influyen en todo el proceso, centrándose solo en un indicador que emana de los estudiantes y obviando los macroindicadores que están detrás.

El modelo presentado da cuenta de estos aspectos y sería interesante replicarlo en otras realidades universitarias para disminuir las tasas de deserción que constituyen un grave problema, para las universidades. La masificación ha restringido sustantivamente la capacidad del docente para desarrollar adecuadamente su tarea con los estudiantes, lo que pone en evidencia la otra cara del problema reconociéndose tres aspectos: la insuficiente preparación de una gran parte del plantel de profesores, la poca dedicación a la docencia y las serias limitaciones en la preparación pedagógica de muchos docentes. Circunstancias que se complican con las modificaciones socioculturales del momento actual.

El estudio indica la urgencia de generar un cuerpo de conocimientos y modelos de gestión de las oficinas de educación médica que, insertas en el plan estratégico, puedan realizar su labor aportando no solo desde la perspectiva microcurricular sino también desde una mirada macrocurricular, otorgando avances significativos en promover procesos formativos de profesionales de la salud más acordes con los nuevos estudiantes y sus actuales requerimientos. Como proyección del modelo de gestión presentado, que incluye docencia, investigación, extensión y gestión, se plantea un cambio desde una oficina de educación a un centro de educación médica y ciencias de la salud.

\section{Referencias}

1. Gimeno J. Comprender y transformar la enseñanza.Madrid: Editorial Morata, duodécima edición; 2008.

2. Díaz-Barriga F. El desarrollo del currículum e innovación curricular: Modelos e investigación en los noventa. Perfiles Educativos 2005; 27 (107): 57-8.

3. World Federation for Medical Education, Estándares globales en Educación médica de la WFME. Revista Educación Médica 2004; 7 (2).

4. Díaz P. Modelo conceptual para la deserción estudiantil universitaria chilena. Estud Pedagog 2008; 34 (2): 65-86.

5. Torres E, Osuna C, Sida P. Reprobación en las carreras del área de ciencias de la salud de la Universidad Autónoma de Baja California, México. Educación y Humanismo 2011; 13 (21): 34-50. 
6. García A. La enseñanza universitaria de la bioética desde una perspectiva interdisciplinaria. En: La Bioética en la formación de recursos humanos de la salud, situación actual y desafíos en el Ecuador. Ministerio de Salud Pública/Comisión de Bioética. Organización Mundial de la Salud. 2009: 61-86. Disponible en: https://www. paho.org/ecu/index.php?option=com_docman\&view=download\&category_slug=desarrollo-de-recursos-humanos-para-la-salud\&alias=104-la-bioetica-en-la-formacion-de-recursos-humanos-de-la-salud\&Itemid=599 [Consultado el 23 de diciembre de 2019].

7. Informe de Desarrollo Social. Ministerio de Desarrollo social. Encuesta CASEN 2018, Disponible en: http:// www.desarrollosocialyfamilia.gob.cl/storage/docs/Informe_de_Desarrollo_Social_2018.pdf. [Consultado el 23 de diciembre de 2019].

8. González L, Uribe D, Estimaciones sobre la "repitencia" y deserción en la educación superior chilena. Consideraciones sobre sus implicaciones. Calidad en la Educación 2002; 17: 75-90.

9. Donoso S, Schiefelbein E. Análisis de los modelos explicativos de retención de estudiantes en la universidad: una visión desde la desigualdad social. Estud Pedagog 2007; 1: 7-27.

10. Stufflebeam D, The CIPP model for program evaluation. In: Evaluation Models. Viewponts on Educational and Human Services Evaluation. Editorial Kluwer Nijhof. Bosto, Mass. USA. 1983.

11. Restrepo B. Aprendizaje basado en problemas (ABP): una innovación didáctica para la enseñanza universitaria. Educación y Educadores 2005; 8: 9-19.

12. Santelices L, Williams C, Soto M, Dougnac A. Impacto de un programa de nivelación de ciencias básicas en estudiantes de primer año de la carrera de medicina. Rev Med Chile 2013; 141: 710-5.

13. Williams C, Cádiz M, Santelices L, Soto M, Dougnac A. Impacto de un programa de Nivelación de Ciencias Básicas en estudiantes de primer año de la carrera de Nutrición y Dietética de la Facultad de Medicina de la Universidad Finis Terrae. Rev Educ Cien Salud 2018; 15 (1): 31-4.

14. Santelices L, Williams C, Soto M, Dougnac A. Evaluación de un programa de perfeccionamiento docente implementado en la Facultad de medicina de la Universidad Finis Terrae. Rev Med Chile 2015; 143: 1152-61.

15. Salas R, Quintana M, Pérez G. Formación basada en competencias en ciencias de la salud. Medisur [online]. 2016; 14 (4): 456-463. Disponible en: http://scielo.sld. $\mathrm{cu} /$ scielo.php? script $=$ sci_arttext\&pid $=\mathrm{S} 1727-897 \mathrm{X}$ 2016000400013\&lng=es\&nrm=iso. ISSN 1727-897X. [Consultado el 16 de diciembre de 2019].

16. Berríos P. Normas y percepciones sobre carrera académica en Chile. Calidad en la Educación 2008; 28: 39-52.

17. Devlin M. Samarawickrema G, The criteria of effective teaching in a changing higher education context. Higher Education Research and Development 2010; 29 (2): 11124. 